\title{
The Angular Diameter and Fundamental Parameters of Sirius A
}

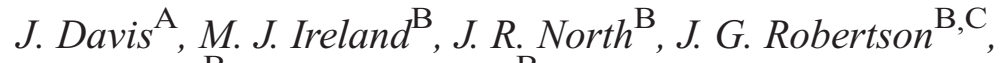 \\ W. J. Tango ${ }^{\mathrm{B}}$, and P. G. Tuthill ${ }^{\mathrm{B}}$ \\ A Deceased. Formerly of Sydney Institute for Astronomy, School of Physics, \\ University of Sydney, NSW 2006, Australia \\ B Sydney Institute for Astronomy, School of Physics, University of Sydney, \\ NSW 2006, Australia \\ ${ }^{\mathrm{C}}$ Corresponding author. Email: Gordon.Robertson@sydney.edu.au
}

Received 2010 April 23, accepted 2010 October 8

\begin{abstract}
The Sydney University Stellar Interferometer (SUSI) has been used to make a new determination of the angular diameter of Sirius A. The observations were made at an effective wavelength of $694.1 \mathrm{~nm}$ and the new value for the limb-darkened angular diameter is $6.048 \pm 0.040$ mas $( \pm 0.66 \%)$. This new result is compared with previous measurements and is found to be in excellent agreement with a conventionally calibrated measurement made with the European Southern Observatory's Very Large Telescope Interferometer (VLTI) at $2.176 \mu \mathrm{m}$ (but not with a second globally calibrated VLTI measurement). A weighted mean of the SUSI and first VLTI results gives the limb-darkened angular diameter of Sirius A as $6.041 \pm 0.017$ mas $( \pm 0.28 \%)$. Combination with the Hipparcos parallax gives the radius equal to $1.713 \pm 0.009 R \odot$. The bolometric flux has been determined from published photometry and spectrophotometry and, combined with the angular diameter, yields the emergent flux at the stellar surface equal to $(5.32 \pm 0.14) \times 10^{8} \mathrm{~W} \mathrm{~m}^{-2}$ and the effective temperature equal to $9845 \pm 64 \mathrm{~K}$. The luminosity is $24.7 \pm 0.7 L_{\odot}$.
\end{abstract}

Keywords: stars: individual ( $\alpha$ CMa, HR 2491) — stars: distances — techniques: interferometric

\section{Introduction}

Sirius A (HR 2491, HD 48915) is the brightest star in the sky $(V=-1.46)$. It has a spectral classification of A1 V and it is also a member of a binary system with a less massive companion Sirius B, first predicted by Bessel (1844), that is now known to be a white dwarf. The angular diameter of Sirius A was first determined by Hanbury Brown \& Twiss (1956) in an experiment to demonstrate the potential of intensity interferometry to overcome the problems of seeing and extreme mechanical stability that had prevented the development of the Michelson interferometer at that time. Hanbury Brown \& Twiss (1958) refined their analysis to give the angular diameter of Sirius A as $7.1 \pm 0.55$ mas (pe). While this value is now only of historical interest, this successful experiment led to the development of the Narrabri Stellar Intensity Interferometer (NSII) (Hanbury Brown et al. 1967). Since the pioneering measurement by Hanbury Brown \& Twiss the angular diameter of Sirius A has been measured with long-baseline optical/infrared interferometers with increasing accuracy. These measurements include those by Hanbury Brown et al. (1974) with the NSII, by Davis \& Tango (1986) with the prototype for the Sydney University Stellar Interferometer (SUSI), by Mozurkewich et al. (2003) with the Mark III interferometer, and by Kervella et al. (2003) and Richichi et al. (2009) with the European Southern Observatory's Very Large Telescope Interferometer (VLTI). It should be noted that in all these measurements the white dwarf companion is too faint to have any influence on the results for the angular size of the primary.

Hayes (1985) reviewed the status of stellar absolute fluxes and energy distributions from 0.32 to $4.0 \mu \mathrm{m}$ and included the energy distribution of Vega (HR 7001, HD 172167) from 330 to $1050 \mathrm{~nm}$. This energy distribution for Vega has been generally accepted as the primary spectrophotometric standard for the visual region of the spectrum but Vega's dust shell creates problems in the infrared. Cohen et al. (1992) have calibrated a model atmosphere for Vega with Hayes' energy distribution and used infrared photometry differentially to establish an absolute scale for a model atmosphere for Sirius A. They derive an angular diameter of 6.04 mas for Sirius A and advocate the use of Sirius A as the primary infrared standard beyond $20 \mu \mathrm{m}$ because of Vega's dust shell. It follows that the angular diameter of Sirius A has assumed particular importance.

The most recent measurements of the angular diameter of Sirius A with the VLTI (Kervella et al. 2003; Richichi, Percheron \& Davis 2009) have by far the smallest uncertainties (by at least a factor of 4) but the values for the angular diameter are larger than the preceding values and the two VLTI values differ by more than twice their standard errors. Because of the importance of the angular diameter of Sirius A we have made an independent measurement with SUSI using the relatively new red beamcombination system (Davis et al. 2007a) with its improved 
calibration and seeing correction techniques compared with the initial blue-sensitive system. In Section 2 the observations and their calibration are described. The detailed analysis of the resulting calibrated values of fringe visibility squared $\left(V^{2}\right)$ is described in Section 3 and the results are compared with previous measurements of the angular diameter of Sirius A in Section 4. Fundamental parameters for Sirius A are derived and presented in Section 5.

\section{The Observations}

The observations were made on 4 nights in March-April 2007 with SUSI (Davis et al. 1999) using the red beamcombination system (Davis et al. 2007a) operating at a nominal wavelength of $700 \mathrm{~nm}$ with an $80 \mathrm{~nm}$ spectral bandwidth. A total of 24 observations, listed in Table 1, were made with baselines of $5,10,15$, and $20 \mathrm{~m}$.

\subsection{Calibration}

Calibration of the observed values of $V^{2}$ is essential to minimise the effects of instrumental visibility losses and

Table 1. Calibrated visibility squared $\left(V_{* \text { cal }}^{2}\right)$ measurements for Sirius as a function of date in 2007. The baseline is the projected baseline. The values of $V_{*_{\text {cal }}}^{2}$ are the values obtained after omitting $\eta \mathrm{CMa}$ from the calibration process (see Section 3 )

\begin{tabular}{lccc}
\hline Date & Zenith angle $(\mathrm{deg})$ & Baseline $(\mathrm{m})$ & $V^{2}{ }^{2} \mathrm{cal}$ \\
\hline March 14 & 20.1 & 14.64 & $0.388 \pm 0.018$ \\
& 22.4 & 14.67 & $0.388 \pm 0.018$ \\
& 24.7 & 14.70 & $0.394 \pm 0.018$ \\
& 27.1 & 14.73 & $0.391 \pm 0.021$ \\
& 29.9 & 14.77 & $0.406 \pm 0.023$ \\
March 15 & 32.7 & 14.81 & $0.375 \pm 0.030$ \\
& 13.6 & 19.44 & $0.156 \pm 0.009$ \\
& 13.8 & 19.44 & $0.152 \pm 0.007$ \\
& 14.5 & 19.45 & $0.163 \pm 0.007$ \\
& 24.2 & 19.59 & $0.146 \pm 0.018$ \\
April 12 & 26.9 & 19.64 & $0.151 \pm 0.016$ \\
& 29.5 & 19.69 & $0.153 \pm 0.018$ \\
& 20.3 & 4.88 & $0.938 \pm 0.025$ \\
& 22.8 & 4.89 & $0.889 \pm 0.026$ \\
& 25.0 & 4.90 & $0.917 \pm 0.025$ \\
April 15 & 27.4 & 4.91 & $0.909 \pm 0.022$ \\
& 30.3 & 4.93 & $0.884 \pm 0.022$ \\
& 35.0 & 4.95 & $0.929 \pm 0.028$ \\
& 38.4 & 4.96 & $0.936 \pm 0.024$ \\
& 41.3 & 4.97 & $0.955 \pm 0.030$ \\
& 36.1 & 9.90 & $0.669 \pm 0.018$ \\
& 39.0 & 9.93 & $0.656 \pm 0.025$ \\
& 41.7 & 9.95 & $0.678 \pm 0.021$ \\
& 47.3 & 9.96 & $0.630 \pm 0.021$ \\
\hline
\end{tabular}

residual seeing effects. Each observation of Sirius was bracketed by observations of two of the three selected calibrators that are listed in Table 2. After the initial analysis involving all three calibrators, which is described here, it was found that $\eta \mathrm{CMa}$ was not a satisfactory calibrator. The discovery and subsequent calibration omitting $\eta \mathrm{CMa}$ is described in Section 3. The angular diameters of all three calibrators were measured with the NSII. They are all small compared with the angular diameter of Sirius A so that the uncertainties in their expected $V^{2}$ values, at the baselines employed, should be essentially negligible compared with the uncertainties in the measured values of $V^{2}$. The effective wavelength of observations for all three calibrators is estimated to be $693 \pm 2 \mathrm{~nm}$ following the procedure described by Davis et al. (2007a). The uniform-disk angular diameters at $693 \mathrm{~nm}$ have been derived from the limb-darkened angular diameters given by Hanbury Brown, Davis \& Allen (1974) using the appropriate correction factors from the tabulation by Davis, Tango \& Booth (2000) which were derived from Kurucz model atmosphere centre-tolimb intensity distributions (Kurucz 1993a, 1993b). The uncertainties in the uniform-disk angular diameters of the calibrators have been taken into account in the analysis of the observational data.

The calibration is achieved using a transfer function ( $T$ ) for each observation of a calibrator defined by

$$
T=\frac{V_{\mathrm{obs}}^{2}}{V_{\mathrm{exp}}^{2}}
$$

where $V_{\text {obs }}^{2}$ is the observed value of $V^{2}$ and $V_{\exp }^{2}$ is the expected value of $V^{2}$ computed from the projected baseline of the observation and the uniform-disk angular diameter.

Details of how the $T$ values have been used are given in Section 3 .

\subsection{Observational Technique}

Details of the standard SUSI observing technique have been described by Davis et al. (2007a). In brief, for the observations of Sirius A and its calibrators, each observation consisted of a set of 1000 scans through the fringe envelope, together with photometric measurements for normalisation purposes. Each observation took approximately 6 minutes so each bracketed observation of Sirius A was completed in $\sim 18$ minutes. Although it was

Table 2. The three calibrators used for the observations of Sirius. All three stars had their angular diameters measured with the Narrabri Stellar Intensity Interferometer (Hanbury Brown, Davis \& Allen 1974). The published limb-darkened angular diameters have been converted to uniform-disk angular diameters using correction factors interpolated from the tabulation of Davis, Tango \& Booth (2000). The angle $\alpha$ is the great-circle distance on the sky of the calibrator from Sirius

\begin{tabular}{lllllr}
\hline HR & Name & Spectral type & $V$ & $\theta_{\text {UD }}(\operatorname{mas})$ & $\alpha(\operatorname{deg})$ \\
\hline 2004 & к Ori & B0.5 Ia & 2.06 & $0.44 \pm 0.03$ & 15.5 \\
2294 & B CMa & B1 II-III & 1.97 & $0.51 \pm 0.03$ & 5.4 \\
2827 & † CMa & B5 I & 2.46 & $0.73 \pm 0.06$ & 15.3 \\
\hline
\end{tabular}


impossible to match the range in hour angle (and hence zenith angle) exactly for the four baselines, they were matched as far as possible and the mean zenith angle of each observation is listed in Table 1. Automatic alignment checks were carried out at intervals of approximately one hour as described by Davis et al. (2007a).

\section{The Analysis}

The observational data were initially processed with the SUSI pipeline as described by Davis et al. (2007a) which outputs a value of $V^{2}$ for each observation, appropriately flux-normalised and seeing-corrected. Subsequent analysis was carried out using an MS EXCEL spreadsheet. The first step was to compute $T$ for each calibrator observation. Next the weighted mean $\bar{T}$ for each pair of bracketing calibrators was found and the intervening value of $V^{2}$ for Sirius A was divided by the mean $T$ to obtain a calibrated value of $V^{2}$ as given by

$$
V_{* \mathrm{cal}}^{2}=\frac{V_{* \mathrm{obs}}^{2}}{\bar{T}}
$$

where $V_{*_{\text {obs }}}^{2}$ is the value of $V^{2}$ for Sirius A from the pipeline and $V_{*_{\text {cal }}}^{2}$ is the calibrated value of $V^{2}$ for Sirius A.

An initial fit to the data to determine the uniform-disk angular diameter as described in Section 3.1 gave a zero baseline value for $V_{* \text { cal }}^{2}$ of $A=1.0348 \pm 0.0094,3.7 \sigma$ greater than the expected value of unity. An investigation was therefore made of the calibrators by inter-comparing their transfer functions. For each pair of calibrators bracketing a Sirius observation their transfer functions $(T)$ were plotted against each other. In the case of the plot for $\beta$ CMa versus $\kappa$ Ori the slope is $1.000 \pm 0.013$ showing that these two calibrators are in good agreement, with the scatter about the fitted line consistent at all four baselines. The corresponding plots of $T$ for $\eta \mathrm{CMa}$ against $\beta \mathrm{CMa}$ and against $\kappa$ Ori on the other hand show a different slope for each baseline, in all cases significantly different from unity. This suggests a previously unsuspected binary nature for $\eta \mathrm{CMa}$. Without a detailed orbit for $\eta \mathrm{CMa}$ it is not possible to use this star as a calibrator. Observations of $\eta \mathrm{CMa}$ have therefore been ignored and the following procedure adopted. Where $\eta \mathrm{CMa}$ was observed, the weighted mean value of $T$ for the $\beta \mathrm{CMa}$ and $\kappa$ Ori on either side of it was adopted. The two intervening values of $V_{* \text { cal }}^{2}$ have the same calibration value of $T$. This approach was possible for all except two Sirius observations at $20 \mathrm{~m}$ and these have been omitted. The intervals between the remaining calibrator observations are increased from $\sim 12$ minutes to $\sim 24$ minutes but this is acceptable.

The calibrated values of $V^{2}$ for Sirius A (i.e. $V_{* \text { cal }}^{2}$ ) are listed in Table 1.

\subsection{The Uniform-Disk Angular Diameter}

The uniform-disk angular diameter is obtained by means of a least-squares fit of the following expression to the calibrated values of $V^{2}$ listed in Table 1

$$
V_{* \mathrm{cal}}^{2}=A\left|\frac{2 J_{1}(x)}{x}\right|^{2}
$$

where $J_{1}(x)$ is a Bessel function, $x=\pi b \theta_{\mathrm{UD}} / \lambda_{\text {eff }}$, with $b$ the projected baseline, $\theta_{\mathrm{UD}}$ the uniform-disk angular diameter, and $\lambda_{\text {eff }}$ the effective wavelength of observation. $A$ is the value of $V^{2}$ obtained by extrapolating the fitted curve to the zero baseline. The fit is therefore a twoparameter fit for the uniform-disk angular diameter $\left(\theta_{\mathrm{UD}}\right)$ and the zero baseline value of $V_{*_{\text {cal }}}^{2}(A)$.

The effective wavelength for observations of mainsequence stars using SUSI's $700 \mathrm{~nm}$ filters has been studied by Davis et al. (2007a), where two methods were used: (i) calculation based on the known filter bandpass, stellar spectral distributions and instrumental sensitivity as a function of wavelength and (ii) measurements based on the fringe frequency derived from laser-calibrated scans. Rather than simply take the value from the tabulation of Davis et al. (2007a), we here make use of the fact that Sirius was one of the stars for which a measurement was made. The measured value was $693.69 \pm 0.34 \mathrm{~nm}$ while the calculated value was $694.5 \mathrm{~nm}$. Owing to the scatter in the measured values of $\lambda_{\text {eff }}$, we err on the side of caution, and adopt a value of $694.1 \mathrm{~nm}$, which is midway between the computed and measured values, but with an uncertainty of $\pm 1.0 \mathrm{~nm}$ to allow for the uncertainties in the measured and computed values for this star.

The relatively broad spectral bandwidth may lead to bandwidth smearing. The resolution of the interferometer will vary across the band and limb-darkening, which is wavelength-dependent, will also vary across the band. These effects have been discussed by Tango \& Davis (2002) and have been investigated specifically for the current observations of Sirius A. Bandwidth smearing is not significant in this case and the conclusion is that equation (3) can be fitted to the observational data using the effective wavelength as discussed above.

A fit of equation (3) to the individual values of $V_{*_{\text {cal }}}^{2}$ in Table 1 gives $A=1.0151 \pm 0.0093( \pm 0.9 \%)$ and $\theta_{\text {UD694.1 }}=5.872 \pm 0.034$ mas $( \pm 0.6 \%)$ with a reduced $\chi^{2}=0.63$. Although the fit gives a zero baseline value of $A$ that is $1.6 \sigma$ greater than the expected value of unity this is regarded as satisfactory in view of the comparison of the two calibrators that were adopted.

The uniform-disk angular diameter, taking the uncertainty in the effective wavelength into account, is $5.872 \pm 0.038$ mas. The fit to all the observational data is shown in Figure 1. As can be seen the fit to all four baselines is excellent.

The eight individual values of $V_{*_{\text {cal }}}^{2}$ for the $20 \mathrm{~m}$ baseline are shown in Figure 2 and show the small scatter in the data.

\subsection{Limb-darkening Correction}

In order to obtain the true, limb-darkened angular diameter a wavelength-dependent correction has to be applied to the uniform-disk angular diameter. Davis, Tango \& Booth (2000) have tabulated correction factors, 
derived from Kurucz model atmosphere centre-to-limb intensity distributions (Kurucz 1993a, 1993b), as a function of wavelength, effective temperature, surface gravity, and $[\mathrm{Fe} / \mathrm{H}]$.

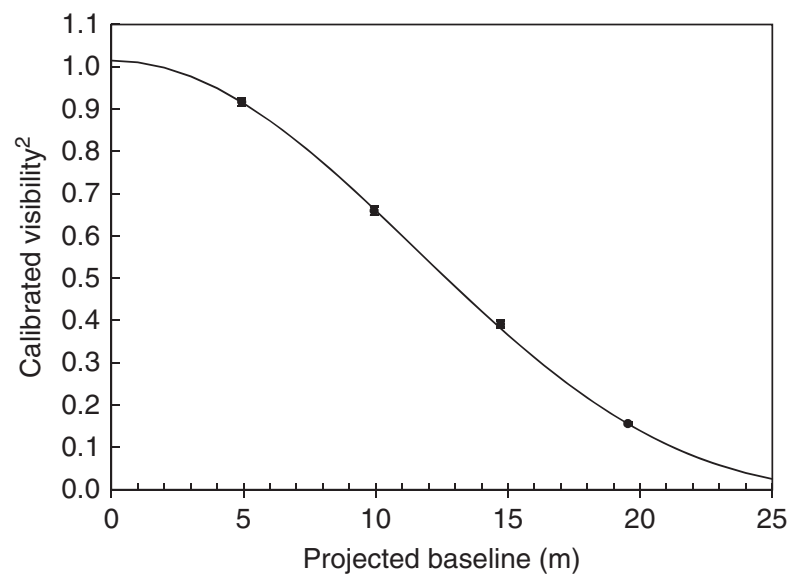

Figure 1 The weighted mean values of calibrated squared visibility at the four baselines used for the observations. The line is the fit to the individual values of observational data listed in Table 1.

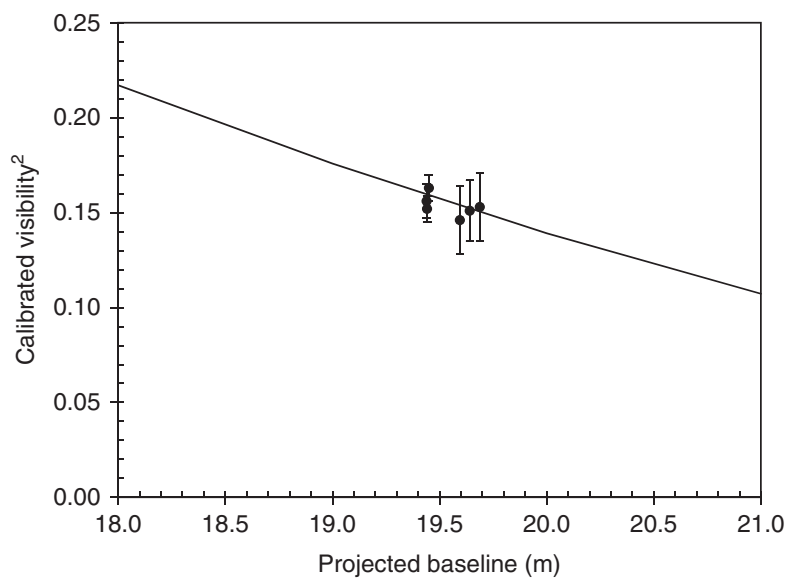

Figure 2 The individual calibrated squared visibilities at the longest baseline. Although all six measurements were made on the same night, the seeing deteriorated significantly between the first three and last three measurements as illustrated by the larger error bars.
For Sirius A the following parameters have been adopted following Cohen et al. (1992): $T_{\text {eff }}=9850 \mathrm{~K}$, $\log g=4.25$, and $[\mathrm{Fe} / \mathrm{H}]=+0.5$. With the effective wavelength of $694.1 \mathrm{~nm}$, interpolation in the Davis, Tango \& Booth (2000) tabulation gives $\rho_{694.1}=1.030$. Although the interpolation itself is accurate to 0.0001 we adopt an uncertainty of 0.001 because the Kurucz models used by Davis, Tango \& Booth (2000) may not be precisely representative of Sirius A.

The limb-darkened angular diameter is therefore $6.048 \pm 0.040$ mas.

\section{The Limb-darkened Angular Diameter of Sirius A}

Table 3 lists the measurements of the angular diameter of Sirius A made to date and Figure 3 plots the tabulated values of limb-darkened angular diameter in order of measurement.

Sirius was a difficult target for the Mark III interferometer because it has a minimum zenith angle of $\sim 51^{\circ}$ at Mount Wilson. Seeing introduces increasing uncertainties in the measurement of $V^{2}$ with zenith angle for amplitude interferometry, particularly in the blue, and this is shown by the larger uncertainty for the $451 \mathrm{~nm}$ measurement compared with that at $800 \mathrm{~nm}$.

The VLTI measurement of Sirius by Kervella et al. (2003) achieved an accuracy of $\pm 0.31 \%$ in spite of using $\theta$ Cen as the sole calibrator for the long baseline $(\sim 62 \mathrm{~m})$ observations. $\theta$ Cen is not an ideal calibrator because it has a relatively large angular diameter and is $\sim 96^{\circ}$ distant from Sirius. However, these disadvantages appear to have been overcome by the fact that the VLTI/VINCI instrumental combination is significantly less prone to seeing effects as a result of the spatial filtering by the optical fiber coupling in VINCI. As will be seen from Table 3 this result and the new SUSI measurement are in excellent agreement.

The VLTI determination of the angular diameter of Sirius by Richichi, Percheron \& Davis (2009) differs from the Kervella et al. (2003) and new SUSI measurements in that it was not a direct measurement based on individually calibrated values of $V^{2}$. It was the result of a global fit

Table 3. Measurements of the limb-darkened angular diameter of Sirius. Entries marked with an asterisk are published uniform-disk angular diameters that have been converted to limb-darkened angular diameters with the limb-darkening factor $\rho_{\lambda}$ interpolated from the tabulation by Davis, Tango \& Booth (2000). In the case of the published Mark III values a separate limb-darkened angular diameter was also included (Mozurkewich et al. 2003)

\begin{tabular}{|c|c|c|c|c|c|c|}
\hline Year & Instrument & $\lambda(\mathrm{nm})$ & $\theta_{\mathrm{UD}}(\mathrm{mas})$ & $\rho_{\lambda}$ & $\theta_{\mathrm{LD}}(\mathrm{mas})$ & Ref. \\
\hline 1958 & NSII prototype & 500 & - & - & $7.1 \pm 0.9$ & 1 \\
\hline 1974 & NSII & 443.0 & $5.60 \pm 0.15$ & 1.052 & $5.89 \pm 0.15$ & 2 \\
\hline 1986 & SUSI prototype * & 441.6 & $5.63 \pm 0.08$ & 1.052 & $5.92 \pm 0.08$ & 3 \\
\hline 2003 & Mark III* & 800 & $5.823 \pm 0.105$ & 1.025 & $5.966 \pm 0.108$ & 4 \\
\hline 2003 & Mark III* & 451 & $5.421 \pm 0.255$ & 1.050 & $5.694 \pm 0.268$ & 4 \\
\hline 2003 & Mark III & 800 & - & - & $5.993 \pm 0.108$ & 4 \\
\hline 2003 & VLTI (single cal) & $\mathrm{K}$ band & - & - & $6.039 \pm 0.019$ & 5 \\
\hline 2009 & VLTI (global cal) & 2181 & $6.030 \pm 0.020$ & 1.0097 & $6.089 \pm 0.020$ & 6 \\
\hline 2010 & SUSI & 694.1 & $5.872 \pm 0.038$ & 1.030 & $6.048 \pm 0.040$ & 7 \\
\hline
\end{tabular}

References : (1) Hanbury Brown \& Twiss (1958); (2) Hanbury Brown, Davis \& Allen (1974); (3) Davis \& Tango (1986); (4) Mozurkewich et al. (2003); (5) Kervella et al. (2003); (6) Richichi, Percheron \& Davis (2009); (7) This work. 


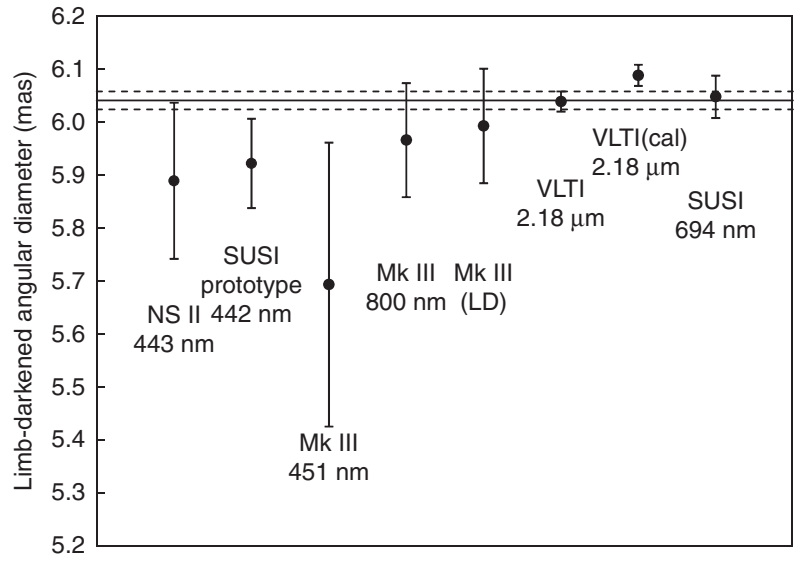

Figure 3 The limb-darkened angular diameter determinations for Sirius listed in Table 3, except for the original historical measurement of Hanbury Brown \& Twiss (1958), in order of publication. The horizontal line is the weighted mean of the VLTI and SUSI values and the dashed lines represent the uncertainty in the mean. The VLTI(cal) value is from Richichi et al. (2009).

to some 700 values of $V^{2}$ determined over 50 nights for 17 stars selected as 'primary' calibrators. The analysis was based on the assumption that the transfer function remained constant during each night and criteria were applied to select good-quality data. In the case of Sirius, 26 separate observations spread over 6 nights were involved in obtaining the result listed in Table 3. The resulting limb-darkened angular diameter is larger than that of Kervella et al. (2003) as noted by Richichi, Percheron \& Davis (2009) and also larger than the SUSI value. It lies more than twice its standard error from both. Although the formal uncertainty is small there are concerns about the convergence of the algorithm used by Richichi, Percheron \& Davis (2009) and the validity of the assumption of a constant transfer function for each night of observation. These limitations were recognised and discussed by Richichi et al. and, while in general their results were in good agreement with the results of other measurements for stars in common, they noted that Sirius was an exception.

It is desirable to combine measurements to determine a weighted mean value for the limb-darkened angular diameter of Sirius but this poses the question of which values to accept. Examination of the values listed in Table 3 shows that the early values, including the Mark III result, have relatively low weights and that they would have little influence on a weighted mean value. The remaining values are the two VLTI results and the new SUSI value. The concerns expressed regarding the Richichi, Percheron \& Davis (2009) VLTI result, and the fact that it lies significantly above the other two values, suggests that the formal uncertainty in this case is underestimated and, furthermore, that some undetermined systematic effect may exist. One possibility would be to arbitrarily increase the uncertainty but, since the other two values are in good agreement, a weighted mean of the Kervella et al. (2003) VLTI and SUSI results has been adopted. The weighted mean of these two results for the limb-darkened angular diameter of Sirius A is $6.041 \pm 0.017$ mas. The value of 6.04 mas determined by Cohen et al. (1992) by spectral radiance calibration is in excellent agreement with the value determined by direct measurement.

\section{The Parameters of Sirius A}

The combination of the limb-darkened angular diameter with the bolometric flux received from a star and with its parallax enables a number of important stellar parameters to be determined. First the bolometric flux received from the star must be established.

\subsection{The Bolometric Flux}

The integrated flux for Sirius was determined by Code et al. (1976) to be $(114.3 \pm 4.4) \times 10^{-9} \mathrm{~W} \mathrm{~m}^{-2}$. However, additional data have become available and improvements in calibration have been achieved since the Code et al. value was published. Following a similar procedure to that employed by Code et al. a new value for the bolometric flux received from Sirius has been established. The spectrum has been divided into four wavelength ranges for this purpose: $0-0.33 \mu \mathrm{m}, 0.33-0.80 \mu \mathrm{m}, 0.80-5.00 \mu \mathrm{m}$, and $5.00-\infty \mu \mathrm{m}$. The flux beyond $5.0 \mu \mathrm{m}$ is $<0.1$ per cent of the total flux for a model stellar atmosphere for Sirius and, as discussed below, allowance is made for it.

It is noted that the $(B-V)$ and $(U-B)$ colours for Sirus are consistent with those of an unreddened star of its spectral classification and no correction for interstellar extinction has been necessary.

\subsubsection{Ultraviolet Flux}

Ultraviolet flux measurements include four photometric measurements made with the TD-1 satellite (Thompson et al. 1978), five photometric measurements made with the OAO-2 satellite (Code, Holm \& Bottemiller 1980), and an OAO-2 spectrum (Code \& Meade 1979). The latter represents by far the most comprehensive flux data for the wavelength range 120 $360 \mathrm{~nm}$, and it is in excellent agreement with the adopted visual fluxes, discussed in the next section, in the overlap region $330-360 \mathrm{~nm}$. In view of this the OAO-2 spectrum has been adopted for the wavelength range 120-330 nm and it has been integrated in three sections: $120-130 \mathrm{~nm}$, $130-180 \mathrm{~nm}$, and $180-330 \mathrm{~nm}$, corresponding to the ranges given by Bless, Code \& Fairchild (1976) with differing calibration uncertainties. The uncertainties for the three ranges have been estimated following the procedure adopted by Code et al. (1976) but, whereas Code et al. made estimates for the hottest stars and applied them to all stars, here the estimates have been made specifically for Sirius A based on the observed flux distribution. In order to estimate the uncertainty in the integrated flux for the band $130-180 \mathrm{~nm}$, the uncertainty in the absolute flux at $130 \mathrm{~nm}$ has been assumed to be $\pm 30 \%$ and, at $180 \mathrm{~nm}, \pm 10 \%$, varying linearly between these limits. For the band $180-330 \mathrm{~nm}$ the uncertainty at $180 \mathrm{~nm}$ has been assumed to be $\pm 10 \%$ and, at $330 \mathrm{~nm}$, $\pm 5 \%$, varying linearly in between. For $120-130 \mathrm{~nm}$ the 
fluxes are uncertain and, following Code et al. (1976), an uncertainty of $\pm 35 \%$ has been adopted. The integrated fluxes for the ultraviolet are: for $120-130 \mathrm{~nm}(0.3 \pm 0.1)$, for $130-180 \mathrm{~nm}(11.0 \pm 1.9)$, and for $180-330 \mathrm{~nm}$ $(23.4 \pm 1.7)$, all $\times 10^{-9} \mathrm{~W} \mathrm{~m}^{-2}$.

\subsubsection{Visual Flux}

Spectrophotometric scans of the visual region of Sirius's spectrum include those by Aller, Faulkner \& Norton (1966) (339.2-586.8 nm), Gutierrez-Moreno, Moreno \& Stock (1968) (339-584 nm), Schild, Peterson \& Oke (1971) (330-605 nm), and Davis \& Webb (1974) (330-808 nm). The scan by Davis \& Webb (1974) has been adopted since it covers the greatest wavelength range. Support for this decision is given by the excellent agreement found for the Davis \& Webb (1974) spectrophotometry, in the case of $\delta$ CMa (Davis et al. 2007b), with that of Kiehling (1987) and the flux distribution in the MILES library of empirical spectra (Sánchez-Blázquez et al. 2006).

Code et al. (1976) used the spectrophotometric calibration of $\alpha$ Lyr (Vega) by Oke \& Schild (1970) to convert the relative spectrophotometry of Davis \& Webb (1974) into a relative absolute flux distribution. Here the more recent spectrophotometric calibration of Vega by Hayes (1985) has been used. Following Code et al. (1976) the resulting relative absolute flux distribution has been scaled by the flux ratio corresponding to the monochromatic magnitude of $\alpha \mathrm{CMa}$ relative to $\mathrm{Vega}$ at $550 \mathrm{~nm}$ $(-1.456)$ measured by Davis (private communication). It has then been converted to fluxes using the value for the flux from Vega at $550.0 \mathrm{~nm}$ of $3.56 \times 10^{-11} \mathrm{~W} \mathrm{~m}^{-2} \mathrm{~nm}^{-1}$ (Megessier 1995).

The spectrophotometric fluxes are continuum fluxes and do not allow for the strong Balmer absorption lines. In order to make allowance for the absorption lines and the lack of measurements between $370 \mathrm{~nm}$ and $403 \mathrm{~nm}$ use has been made of a model atmosphere generated specifically for Sirius by R. Kurucz. The model temperature of $9850 \mathrm{~K}$ is essentially the same as that derived in this work (see Section 5.2) and is therefore appropriate for this purpose. The model flux was integrated for the interval $330-800 \mathrm{~nm}$ and also for the model with the lines replaced by continuum interpolated from each side of the lines. The ratio of flux with the lines to the flux without the lines was found to be 0.9534 . The empirical flux distribution was also integrated with the addition of three points in the $370-403 \mathrm{~nm}$ interval derived from the smoothed model flux distribution to give an integrated flux for $330-800 \mathrm{~nm}$ equal to $67.26 \times 10^{-9} \mathrm{~W} \mathrm{~m}^{-2}$ which, after correction for the lines, becomes $64.13 \times 10^{-9} \mathrm{~W} \mathrm{~m}^{-2}$.

The uncertainty in the visual flux, based on the analysis carried out for $\delta \mathrm{CMa}$ (Davis et al. 2007b), suggests that the uncertainty in the relative absolute flux distribution is at the $1 \%$ level, the uncertainty in the monochromatic difference used for scaling the Sirius flux distribution is estimated to be $\sim 1 \%$, and Megessier (1995) claims $\pm 0.7 \%$ for the flux calibration at $550 \mathrm{~nm}$. These uncertainties are independent and combining them quadratically gives a resultant
Table 4. Adopted calibrated photometric IR flux values for Sirius from the literature. The units for wavelength $(\lambda)$ are $\mu \mathrm{m}$ and for the flux $10^{-9} \mathrm{~W} \mathrm{~m}^{-2} \mu \mathrm{m}^{-1}$

\begin{tabular}{lllll}
\hline Band & $\lambda$ & Flux & Source & Calibration \\
\hline$I$ & 0.798 & 40.9 & 1 & $\mathrm{a}$ \\
$I$ & 0.90 & 31.0 & 2 & $\mathrm{~b}$ \\
$J$ & 1.25 & 10.8 & $2,3,4$ & $\mathrm{c}, \mathrm{a}, \mathrm{a}$ \\
$H$ & 1.65 & 3.90 & 3,4 & $\mathrm{a}$ \\
$K$ & 2.2 & 1.38 & $2,3,4$ & $\mathrm{c}, \mathrm{a}, \mathrm{a}$ \\
$L$ & 3.4 & 0.27 & 2 & $\mathrm{~b}$ \\
$L$ & 3.7 & 0.205 & 3,4 & $\mathrm{c}$ \\
$M$ & 4.8 & 0.076 & 3 & $\mathrm{~d}$ \\
\hline
\end{tabular}

Source references: (1) Cousins (1980); (2) Johnson et al. (1966); (3) Engels et al. (1981); (4) Glass (1974).

Calibration references: (a) Bessell, Castelli \& Plez (1998); (b) Johnson (1966); (c) Megessier (1995); (d) Wamsteker (1981).

uncertainty of $\pm 1.6 \%$. In addition there is an uncertainty associated with the use of the theoretical model atmosphere and we adopt a final uncertainty of $\pm 2.0 \%$. This is an improvement on the uncertainty of $\pm 4 \%$ adopted by Code et al. (1976) but is based on evidence that the spectrophotometric scan of Davis \& Webb (1974) is better than thought by Code et al., the improved Vega calibration by Hayes (1985), and the improved calibration by Megessier (1995). The flux for the wavelength range $330-800 \mathrm{~nm}$ is $(64.1 \pm 1.3) \times 10^{-9} \mathrm{~W} \mathrm{~m}^{-2}$.

\subsubsection{Infrared Flux}

The fluxes for the wavelength range $0.80-5.00 \mu \mathrm{m}$ have been obtained from the $I, J, H, K, L$, and $M$ broadband photometry of Johnson et al. (1966) $(I, J, K, L)$, Cousins (1980) (I), Engels et al. (1981) $(J, H, K, L, M)$, and Glass (1974) $(J, H, K, L)$. Flux calibrations by Johnson (1966), Bessell, Castelli \& Plez (1998), Megessier (1995), and Wamsteker (1981) have been used to convert the photometric magnitudes to fluxes. Multiple measurements for the same band have been averaged and the adopted fluxes are given in Table 4.

The data in Table 4 were plotted against wavelength and a smooth curve drawn through them. The extended curve at the short-wavelength end joined smoothly to the visual fluxes in the $0.7-0.81 \mu \mathrm{m}$ range and was also in agreement with the 13-colour fluxes of Johnson \& Mitchell (1975) in the range $0.724-1.108 \mu \mathrm{m}$. The area under the curve from $0.80 \mu \mathrm{m}$ to $5.0 \mu \mathrm{m}$ was integrated to give the continuum flux for this interval equal to $15.51 \times 10^{-9} \mathrm{~W} \mathrm{~m}^{-2}$. As for the visual region the Kurucz model was integrated with the absorption lines and with the lines replaced by continuum interpolated from each side of the lines. The ratio of flux with the lines to the flux without the lines was found to be 0.9797. The integrated empirical flux, after correction for the lines, becomes $15.20 \times 10^{-9} \mathrm{~W} \mathrm{~m}^{-2}$.

The uncertainties in the data in Table 4 have been computed from the published uncertainties in the photometry and in the calibrations. The average uncertainty across the $2.0-5.0 \mu \mathrm{m}$ band was found to be $\pm 4.1 \%$ and this has been adopted as the uncertainty in the integrated 
Table 5. Integrated fluxes for Sirius in each spectral band and the resulting bolometric flux. The units for the wavelength bands $(\lambda \lambda)$ are $\mu \mathrm{m}$ and for the flux and its uncertainty $(\sigma)$ they are $10^{-9} \mathrm{~W} \mathrm{~m}^{-2}$

\begin{tabular}{lcrc}
\hline Range & \multicolumn{1}{c}{$\lambda \lambda$} & Flux & $\pm \sigma$ \\
\hline UV & $0.12-0.13$ & 0.3 & 0.1 \\
UV & $0.13-0.18$ & 11.0 & 1.9 \\
UV & $0.18-0.33$ & 23.4 & 1.7 \\
Visual & $0.33-0.80$ & 64.1 & 1.3 \\
IR & $0.80-5.00$ & 15.2 & 0.6 \\
Far IR & $>5.00$ & 0.1 & \\
\hline Bolometric flux $(f)$ & 114.1 & 2.9 & \\
\hline
\end{tabular}

flux. The integrated flux is therefore $(15.2 \pm 0.6) \times$ $10^{-9} \mathrm{~W} \mathrm{~m}^{-2}$.

\subsubsection{The Residual Flux for $\lambda>5.0 \mu \mathrm{m}$}

As mentioned in Section 5.1 the flux from Sirius for $\lambda>5.0 \mu \mathrm{m}$ is small. The flux longward of $5 \mu \mathrm{m}$ to $297 \mu \mathrm{m}$ for the Kurucz model atmosphere for Sirius has been integrated and is $0.09 \%$ of the total flux for the model. In the case of a black body similar integrations give $0.11 \%$ of the total flux longward of $5.0 \mu \mathrm{m}$. Since this is such a small fraction of the total flux the IRAS point-source fluxes $(\geq 12 \mu \mathrm{m})$ and the low-resolution scan data have not been considered. The flux longward of $5.0 \mu \mathrm{m}$ has been taken to be $0.10 \pm 0.01 \%$ of the total flux or $(0.11 \pm 0.01) \times 10^{-9} \mathrm{~W} \mathrm{~m}^{-2}$.

\subsubsection{The Total Flux}

The fluxes for the four wavelength ranges considered are listed in Table 5 together with the bolometric flux (f), the total flux received from Sirius, which is equal to $(114.1 \pm 2.9) \times 10^{-9} \mathrm{~W} \mathrm{~m}^{-2}$. The bolometric flux is close to the value of $(114.3 \pm 4.4) \times 10^{-9} \mathrm{~W} \mathrm{~m}^{-2}$ found by Code et al. (1976) but is more accurate due to improvements in the flux calibrations. The flux values used in the UV, visual and IR spectral regions are plotted in Figure 4 together with the flux distribution for the Kurucz model atmosphere $\left(T_{\text {eff }}=9850 \mathrm{~K}, \log g=4.25\right.$, and $[\mathrm{Fe} / \mathrm{H}]=$ $+0.5)$. It is stressed that the integrated empirical fluxes are essentially independent of the model except for corrections for absorption lines not included in the photometric and spectrophotometric spectral bands which amount to a total of $2.9 \%$ of the total flux. The model flux distribution is that fitted to Sirius fluxes, derived from differential Sirius/Vega photometry, by Cohen et al. (1992). The model fluxes are independent of the fluxes determined here and have been included in the figure to illustrate the excellent agreement between the current work and that of Cohen et al.

\subsection{The Emergent Flux and Effective Temperature}

The emergent flux at the stellar surface $(\mathcal{F})$ and the effective temperature $\left(T_{\text {eff }}\right)$ of a star are related by

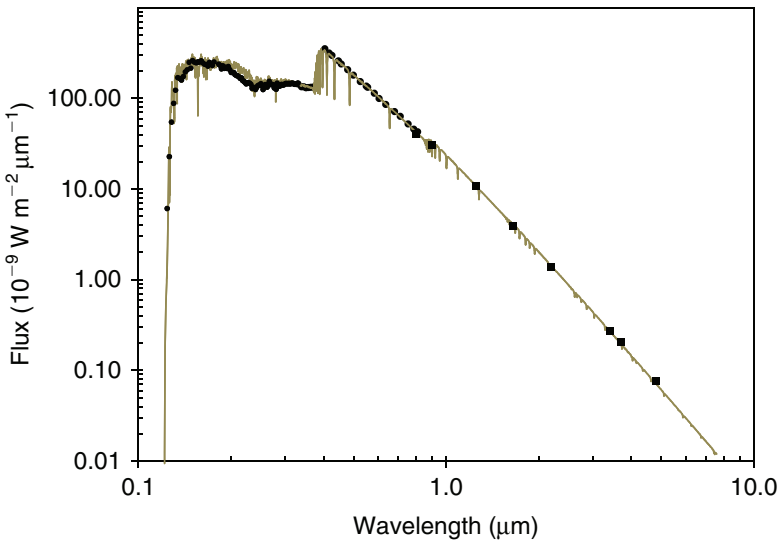

Figure 4 The flux distribution for Sirius from the ultraviolet to the mid-infrared. Key: Filled circles - OAO-2 ultraviolet fluxes (Code \& Meade 1979); open circles — visual fluxes (Davis \& Webb 1974); filled squares - infrared fluxes from Table 4; grey line — Kurucz model atmosphere fluxes (Cohen, private communication). The model fluxes are not a fit to the empirical fluxes.

$$
\sigma T_{\text {eff }}=\mathcal{F}=\frac{4 f}{\theta_{\mathrm{LD}}^{2}}
$$

where $\sigma$ is the Stefan-Boltzmann radiation constant.

Substituting the bolometric flux and limb-darkened angular diameter determined above into equation (4) gives the emergent flux at the stellar surface $\mathcal{F}=(5.32 \pm$ $0.14) \times 10^{8} \mathrm{~W} \mathrm{~m}^{-2}$ and the effective temperature $T_{\text {eff }}=$ $9842 \pm 64 \mathrm{~K}$.

The literature contains many estimates of the effective temperature of Sirius but the only previous determination based on a measured angular diameter and empirically determined flux distribution is that of Code et al. (1976). They obtained $9970 \pm 160 \mathrm{~K}$ which lies within one standard deviation from the result of significantly improved accuracy presented here.

The Kurucz model atmosphere for Sirius which Cohen et al. (1992) used in their approach to absolute stellar calibration had an effective temperature of $9850 \mathrm{~K}$ in remarkable agreement with the independent value established here. Although use was made of this model atmosphere in making allowance for absorption lines it is stressed that the current work is essentially independent of theoretical models. It therefore provides an independent check on the effective temperature and angular diameter determined by Cohen et al. (1992). The agreement for both quantities is remarkable with the present work providing results of significantly improved accuracy.

\subsection{The Radius and Luminosity}

The angular diameter and bolometric flux can be combined with the parallax to determine the stellar radius and the stellar luminosity respectively. Combining the limbdarkened angular diameter with the Hipparcos parallax (van Leeuwen 2007) of $379.21 \pm 1.58$ mas gives the radius of Sirius A as $R / R_{\odot}=1.713 \pm 0.009$. 
Table 6. The parameters of Sirius A. $\theta_{\mathrm{LD}}$ is the limb-darkened angular diameter, $\pi_{\mathrm{p}}$ is the parallax, $f$ is the bolometric flux received from the star, $\mathcal{F}$ the emergent flux at the stellar surface, $T_{\text {eff }}$ the effective temperature, $R / R_{\odot}$ the radius in solar radii and $L / L_{\odot}$ is the luminosity in solar luminosities

\begin{tabular}{lcll}
\hline Parameter & \multicolumn{1}{c}{ Value } & $\pm \%$ & Ref. \\
\hline$\theta_{\mathrm{LD}}(\mathrm{mas})$ & $6.041 \pm 0.017$ & 0.27 & 1 \\
$\pi_{\mathrm{p}}(\mathrm{mas})$ & $379.21 \pm 1.58$ & 0.42 & 2 \\
$f\left(\mathrm{~W} \mathrm{~m}^{-2}\right)$ & $(1.141 \pm 0.029) \times 10^{-7}$ & 2.5 & 1 \\
$\mathcal{F}\left(\mathrm{W} \mathrm{m}^{-2}\right)$ & $(5.32 \pm 0.14) \times 10^{8}$ & 2.6 & 3 \\
$T_{\text {eff }}(\mathrm{K})$ & $9842 \pm 64$ & 0.65 & 3 \\
$R / R_{\odot}$ & $1.713 \pm 0.009$ & 0.5 & 3 \\
$L / L \odot$ & $24.7 \pm 0.7$ & 2.8 & 3 \\
\hline
\end{tabular}

References: (1) This work; (2) van Leeuwen (2007); (3) Derived.

The luminosity is given by

$$
L=4 \pi f \frac{C^{2}}{\pi_{\mathrm{p}}^{2}}
$$

where $C$ is the conversion from parsecs to metres. Substitution leads to $L / L_{\odot}=24.7 \pm 0.7$.

The basic data and derived parameters for Sirius are listed in Table 6.

\section{Summary}

A new accurate limb-darkened angular diameter for Sirius A has been determined which is in excellent agreement with and confirms the ESO/VLTI measurement by Kervella et al. (2003). The weighted mean of the two determinations is $\theta_{\mathrm{LD}}=6.041 \pm 0.017 \mathrm{mas}( \pm 0.27 \%)$. An accurate value for the bolometric flux has been computed from flux-calibrated photometry and spectrophotometry. Combination of the angular diameter with the bolometric flux and the revised Hipparcos parallax has led to improved values for the emergent flux at the stellar surface, the effective temperature, the radius and the luminosity. It is noted that the accuracies of the emergent flux, effective temperature and luminosity are limited by the accuracy of the calibration of the photometric fluxes.

The effective temperature and angular diameter are in excellent agreement with those derived by Cohen et al. (1992) through spectral irradiance calibration in the infrared.

The accurate value for the angular diameter of Sirius A is important for its role as a primary infrared standard beyond $20 \mu \mathrm{m}$ as advocated by Cohen et al. (1992). In addition the improved values and accuracy of radius, effective temperature and luminosity will provide tighter constraints on the evolutionary status of Sirius A than has been possible to date.

\section{Acknowledgments}

The SUSI programme is funded jointly by the Australian Research Council and the University of Sydney. Martin Cohen kindly provided the flux distribution for the model atmosphere for Sirius that Bob Kurucz had computed for him. This work has made use of the SIMBAD database, operated by CDS, Strasbourg, France and VizieR (Ochsenbein, Bauer \& Marcout 2000).

\section{References}

Aller, L. H., Faulkner, D. J. \& Norton, R. H., 1966, ApJ, 144, 1073 Bessel, F. W., 1844, MNRAS, 6, 136

Bessell, M. S., Castelli, F. \& Plez, B., 1998, A\&A, 333, 231

Bless, R. C., Code, A. D. \& Fairchild, E. T., 1976, ApJ, 203, 410

Code, A. D., Davis, J., Bless, R. C. \& Hanbury Brown, R., 1976, ApJ, 203, 417

Code, A. D., Holm, A. V. \& Bottemiller, R. L., 1980, ApJS, 43, 501

Code, A. D. \& Meade, M. R., 1979, ApJS, 39, 195

Cohen, M., Walker, R. G., Barlow, M. J. \& Deacon, J. R., 1992, AJ, 104,1650

Cousins, A. W., 1980, SAAO Circ., 1, 234

Davis, J. \& Tango, W. J., 1986, Nature, 323, 234

Davis, J. \& Webb, R. J., 1974, MNRAS, 168, 163

Davis, J., Tango, W. J. \& Booth, A. J., 2000, MNRAS, 318, 387

Davis, J., Tango, W. J., Booth, A. J., ten Brummelaar, T. A., Minard, R. A. \& Owens, S. M., 1999, MNRAS, 303, 773

Davis, J., et al., 2007a, PASA, 24, 138

Davis, J., et al., 2007b, PASA, 24, 151

Engels, D., Sherwood, W. A., Wamsteker, W. \& Schultz, G. V., 1981, A\&A Supp., 45, 5

Glass, I. S., 1974, MNASSA, 33, 53

Gutierrez-Moreno, A., Moreno, H. \& Stock, J., 1968, Univ. Chile Dept. Astr. Pub., 8, 127

Hanbury Brown, R. \& Twiss, R. Q., 1956, Nature, 178, 1046

Hanbury Brown, R. \& Twiss, R. Q., 1958, Proc.Roy.Soc. (London), A, 248, 222

Hanbury Brown, R., Davis, J. \& Allen, L. R., 1967, MNRAS, 137, 375

Hanbury Brown, R., Davis, J. \& Allen, L. R., 1974, MNRAS, 167,121

Hayes, D. S., 1985, in IAU Symp. 111, Calibration of Fundamental Stellar Quantities, Eds. Hayes, D. S. Pasinetti, L. E. \& Davis Philip, A. G. (Dordrecht, Reidel), 225

Johnson, H. L., 1966, ARA\&A, 4, 193

Johnson, H. L. \& Mitchell, R. I., 1975, Rev. Mex. Astron. Astrofis., 1,299

Johnson, H. L., Mitchell, R. I., Iriarte, B. \& Wisniewski, W. Z., 1966, Comm. Lunar and Plan. Lab., 4, 99

Kervella, P., Thévenin, F., Morel, P., Bordé, P. \& Di Folco, E., 2003 A\&A, 408, 681

Kiehling, R., 1987, A\&AS, 69, 465

Kurucz, R. L., 1993a, Limbdarkening for $2 \mathrm{~km} \mathrm{~s}^{-1}$ grid (No. 13): $[+1.0]$ to $[-1.0]$. Kurucz CD-ROM No. 16

Kurucz, R. L., 1993b, Limbdarkening for $2 \mathrm{~km} \mathrm{~s}^{-1}$ grid (No. 13): $[+0.0]$ to $[-5.0]$. Kurucz CD-ROM No. 17

Mégessier, C., 1995, A\&A, 296, 771

Mozurkewich, D., et al., 2003, AJ, 126, 2502

Ochsenbein, F., Bauer, P. \& Marcout, J., 2000, A\&AS, 143, 221

Oke, J. B. \& Schild, R. E., 1970, ApJ, 161, 1015

Richichi, A., Percheron, I. \& Davis, J., 2009, MNRAS, 399, 399

Sánchez-Blázquez et al., 2006, MNRAS, 371, 703

Schild, R., Peterson, D. M. \& Oke, J. B., 1971, ApJ, 166, 95

Tango, W. J. \& Davis, J., 2002, MNRAS, 333, 642

Thompson, G. I., Nandy, K., Jamar, C., Monfils, A., Houziaux, L., Carnochan, D. J. \& Wilson, R., 1978, Catalogue of stellar ultraviolet fluxes. A compilation of absolute stellar fluxes measured by the Sky Survey Telescope (S2/68) aboard the ESRO satellite TD-1, 1978csuf.book, unknown

van Leeuwen, F., 2007, Hipparcos, the new Reduction of the Raw Data (Springer)

Wamsteker, W., 1981, A\&A, 97, 329 